\title{
A dynamic approach to quadrilateral definitions
}

\author{
Rajendran Govender \\ University of the North \\ email: rajengovender@absamail.co.za \\ Michael de Villiers \\ University of Durban-Westville \\ email: profmd@mweb.co.za
}

This study examined 18 prospective secondary mathematics teachers' understanding of the nature of definitions, and their use of the dynamic geometry software Sketchpad to improve not only their understanding of definitions but also their ability to define geometric concepts themselves. Results indicated that the evaluation of definitions by accurate construction and measurement enabled students to achieve a better understanding of necessary and sufficient conditions, as well as the ability to more readily find counter-examples, and to recognise and improve on uneconomical definitions.

\section{Introduction}

Research by Linchevsky, Vinner \& Karsenty (1992), among others, on definitions in mathematics, has indicated that many student teachers do not understand that definitions in geometry have to be economical (contain no superfluous information) and that they are arbitrary (in the sense that several alternative definitions may exist). It is plausible to conjecture that this is probably due to their past school experiences where definitions were largely supplied to them directly. It would appear that in order to increase future teachers' understanding of geometric definitions, and of the concepts to which they relate, it is essential to engage them at some stage in the process of defining geometric concepts.

The research reported here concentrated mainly on student teachers' (prospective mathematics teachers) understanding of the nature of definitions and the development of their ability to evaluate and formulate definitions in a Sketchpad context (see Govender, 2002). Sketchpad was used to expose the students to the process of defining as a creative activity in which students can be fully involved, rather than perpetuating the view of definition as an imposed body of knowledge immune to any change or development. The following research questions were addressed:
- What prior understanding of the nature of definitions do student teachers have before being engaged in a process of formulating some definitions for themselves?

- To what extent does the student teachers' understanding of the nature of definitions change while involved in a process of evaluating definitions by means of construction, measurement and dragging within a Sketchpad context?

- How competent are the student teachers in evaluating other definitions after being engaged in the preceding process?

\section{Definitions}

Because student teachers often meet mathematics structured only as in condensed formal mathematics textbooks, their learning takes place within this structure. The textbooks used in schools give concisely expressed definitions, and this has an effect both on how our students view definitions and how teachers teach. However, this structured approach can easily lead to a common but false perception that there is only one (correct) definition for each defined object in mathematics. The fact that several different (correct) definitions may exist for a particular concept is seldom addressed in such a structured, pre-packaged approach. 
A further misconception that can easily develop from a traditionally axiomatically structured approach is that mathematics always starts with definitions, which can give the false impression that definitions of mathematical objects are given a priori in nature. In such a structured approach, students are not brought to realise that definitions do not exist independently of human experience in some "ideal" Platonistic world, so that all we can do is to "discover" them. The fact that definitions are not discoveries, but human "inventions" for the main purpose of accurate mathematical communication, is therefore not addressed.

Fortunately, the National Curriculum Statement Grades 10-12 (Schools) lays a foundation for the use of technology such as dynamic geometry software for exploration and investigation. In particular, the grade 10 learners ought to explore necessary and sufficient conditions for the various quadrilaterals and investigate ways of defining various polygons. Furthermore, according to the new FET curriculum, grade 10 learners ought to realise that definitions are not absolute but fixed on the basis of principles that will result in conciseness and efficiency. Definitions, axioms and preceding theorems thus serve as starting points for deductive arguments in the expansion of the axiomatic system. For the quadrilaterals, decisions as to which system of definitions to use can depend on either a partition or inclusiveness approach, although the latter is favoured in the interests of efficiency. Learners need to be made aware of these factors in determining definitions.

Definitions are important in mathematics, but they ought to evolve naturally from previous knowledge, models or real experiences that the child can relate to. Otherwise, confusion reigns. Mathematical definitions are generally very concise, contain mathematical terms, and require an immediate synthesis of the information if understanding is to result. However, although research confirms that most children cannot operate on an abstract or formal level until junior high school, we find many textbooks and teachers presenting formal definitions to children before they reach junior high school. In fact, learners' spatial thinking needs to be relatively mature (welldeveloped) before they are able to decide what the definition of a rhombus (for example) should be. Indeed, according to the Van Hiele theory, they need to be at least at Van Hiele Level 3 (see Burger \& Shaughnessy, 1986; De Villiers, 1997).

\section{The van Hiele Theory}

The Van Hiele model has important implications for the teaching of geometry. It can be used firstly to guide students to achieve a more sophisticated level of geometric thinking, and secondly to assess students' abilities. The most obvious characteristic of the theory is the distinction of five discrete thought levels in the development of students' understanding of geometry. The levels, labelled "visualization (recognition)," "analysis," "informal deduction (ordering)," "deduction" and "rigor" describe characteristics of the thinking process. Assisted by appropriate instructional experiences, the model asserts that the learner moves sequentially from the initial, or basic level (visualization), where space is simply observed and the properties of figures are not explicitly recognised, through the sequence listed above to the highest level (rigor), which is concerned with formal abstract aspects of deduction. Few students are exposed to, or reach, the latter level. According to this model, the learner cannot achieve a specific level of thinking without having passed through previous levels.

According to the Van Hiele theory, the main reason the traditional geometry curriculum fails is that it is presented at a higher level than those at which students are operating; in other words, students cannot understand the teacher nor can the teacher understand why they cannot understand. The general characteristics of the first four levels, the ones commonly displayed by secondary students and most relevant to this study, are given:

Level 1: Recognition: Students visually recognise figures by their global appearance. They recognise triangles, squares, parallelograms and so forth by their shape, but they do not explicitly identify the properties of these figures.

Level 2: Analysis: Students start analyzing the properties of figures and learn the appropriate technical terminology for describing them, but they do not interrelate figures or properties of figures.

Level 3: Ordering: Students logically order the properties of figures by short chains of deductions and understand the relationships between figures (for example, class inclusions).

Level 4: Deduction: Students start developing longer sequences of statements and begin to understand the 
significance of deduction and the role of axioms, theorems and proofs.

The Van Hiele theory is a useful framework for interpreting and analysing student teachers' levels of understanding for the following reasons:

- It seeks to explain not only why students have trouble in learning but also what could be done to remove these stumbling blocks.

- The differences between the levels can be projected in terms of the objects and structure of thought at each level (see Fuys et al., 1986).

- Each level is characterised by a series of specific actions/performances. For example, by using task-based interviews Burger and Shaughnessy (1986) identified more fully what students do at the first four levels. Due to space limitations, we shall only discuss Level 3.

\section{$\underline{\text { Level } 3}$}

- Formulate economical, correct definitions for figures.

- Are able to transform incomplete definitions into complete definitions and more spontaneously accept and use definitions for new concepts.

- Accept different equivalent definitions for the same concept.

- Classify figures hierarchically; for example, quadrilaterals.

- Explicitly use the logical form if.... then to formulate and handle conjectures, and implicitly use logical rules such as modus ponens.

- Are uncertain and lack understanding regarding the functions of axioms, definitions and proof.

- Students attempting definitions of concepts would be influenced by their level of understanding. For example, students who are still at van Hiele Level 1 tend to give visual definitions, e.g. a rectangle which looks like this (draws or identifies a quadrilateral with all angles 90 degrees and two long and two short sides), while those students at van Hiele Level 2 tend to give correct, uneconomical definitions (eg. a rectangle is a quadrilateral with opposite sides parallel and equal, all angles 90 degrees, equal diagonals, half-turn- symmetry, two axes of symmetry through opposite sides, two long and two short sides, and so on.). Students at van Hiele level 3 tend to give correct, economical definitions, e.g. a rectangle is a quadrilateral with two axes of symmetry through opposite sides. (compare De Villiers, 1997: 15-17).

\section{Research Design}

\section{The target population}

The target population of this study was the 18 Mathematics Education 3 students at Umbumbulu College of Education (UCE), which is located approximately $15 \mathrm{~km}$ south of the Durban International Airport, in the Kwa Makutha Township. UCE is a three year teacher college which gives students a professional teacher's diploma. The college prepares its students for teaching at both the junior and senior secondary level. Hence, their mathematical preparation is less than what a regular mathematics student gets at a regular university. On the other hand, there is a lot of emphasis on didactics and pedagogy at the teacher training college.

\section{Characteristics of the subjects (sample) \\ [Backgound of Students]}

Quadrilaterals like the parallelogram, square, rectangle, rhombus and kite are taught in all schools in South Africa. All the students had done mathematics up to matric level at school. Many of them had attempted standard grade mathematics and obtained poor passes. A few had attempted higher grade mathematics and also performed poorly (see Table 3.1). At college the students in the Mathematics Method 1 and 2 Courses revisited many of the topics from the junior secondary and senior secondary mathematics school syllabi. Their first and second year Mathematics Education courses included many of the learning theories and other associated topics such as mathematical thinking; cooperative learning; investigative mathematics; OBE; and Assessment. In the final year of study a great deal of time was allocated to calculus. These students were expected to be conversant with most of the aspects associated with quadrilaterals, in particular the properties of rhombuses, the definition of a rhombus and the associated theorems.

All students were black and had only attended schools for blacks in their previous classes. There were 10 males and 8 females in the given sample. Table 1 shows: 
- the gender distribution in the sample

- age of the respective students

- the students' mathematics symbols in the senior certificate examination and the grades on which the subject was written (higher grade or standard grade)

- the students' second year college exam mathematics content results.

The overall academic performance for each student in the senior certificate examination is poor. The overall academic performance of most of the students in the second year college exam is clearly below average.

The students were preparing themselves to write their final college exit exam. They were all willing participants since they all wanted to become computer literate and also wanted to understand the topic better. The topic is not in the college syllabus and the researcher promised the subjects that he would make them computer literate and issue a certificate of participation to each student.

Many of the students were computer illiterate. During the afternoons and during the mathematics method period the students were exposed to a basic computer literacy programme. The students had no prior exposure to Sketchpad but through organised planning they were brought together in the afternoons in order to familiarise them with the general use and application of the computer software - Sketchpad. Furthermore, they had not been previously exposed to the process of defining. Before engaging them in defining rhombi, the students had to be given some knowledge of the properties of rhombuses and associated theorems. This was well within the capabilities of the students.

\begin{tabular}{|l|c|c|c|c|c|c|}
\hline Name & Age & Gender & $\begin{array}{c}\text { Mathematics } \\
\text { Higher Grade }\end{array}$ & $\begin{array}{c}\text { Mathematics } \\
\text { Standard Grade }\end{array}$ & $\begin{array}{c}\text { Second Year } \\
\text { Final }\end{array}$ & $\begin{array}{c}\text { Second Year } \\
\text { Supplementary }\end{array}$ \\
\hline Buzani & 22 & F & & F & $40 \%$ & $51 \%$ \\
\hline Ephraim & 23 & M & & F & $42 \%$ & $39 \%$ \\
\hline Innocent & 21 & M & & F & $43 \%$ & $44 \%$ \\
\hline Jwara & 20 & M & F & & $58 \%$ & \\
\hline Khethu & 20 & M & & E & $54 \%$ & \\
\hline Letha & 21 & M & & E & $53 \%$ & \\
\hline Mathunzi & 23 & M & & E & $59 \%$ & \\
\hline Mthembu & 23 & F & & E & $61 \%$ & \\
\hline Ntombi & 22 & F & F & & $56 \%$ & \\
\hline Owen & 21 & M & E & & $63 \%$ & \\
\hline Petros & 23 & M & & F & $40 \%$ & $47 \%$ \\
\hline Sanele & 22 & M & & E & $50 \%$ & \\
\hline Siboniso & 21 & M & & E & $59 \%$ & \\
\hline Sifiso & 23 & M & & E & $62 \%$ & \\
\hline Siyand & 23 & M & & F & $43 \%$ & $50 \%$ \\
\hline Thandiwe & 23 & F & & E & $52 \%$ & \\
\hline Xolis;e & 23 & F & & $55 \%$ & \\
\hline Fikile & 23 & F & & $51 \%$ & \\
\hline Table & Partipants & & & \\
\hline
\end{tabular}

Table 1: Participants in the study

\section{Research Approach}

The Developmental Research Approach of the Freudenthal Institute at Utrecht University in the Netherlands was used (Gravemeijer, 1994). Basically this approach involves the designing, assessment and consequent redesign of curricula for learning and teaching mathematics. Use of activities from De Villiers (1999) was made in this study, as well as the associated theoretical framework in relation to different functions of proof in mathematics. 
The Van Hiele theory of learning geometry also provided a useful conceptual framework for interpreting and analysing the student teachers' levels of understanding (see Burger \& Shaughnessy, 1986).

\section{Data Collection}

Both qualitative and quantitative data were collected between February and May 2000 from 18 students, largely by means of one-to-one task-based interviews. The rhombus was chosen for the task-based activity. The researcher chose this quadrilateral because in his experience as a teacher he had found that learners at schools have a lot of difficulty in understanding this concept, and in meaningfully applying the knowledge gained about a rhombus. They also have difficulty in "connecting" the knowledge gained to other quadrilaterals.

The collection of data was of a dual nature, i.e. there were written responses to some questions and verbal responses to others. In addition, there were follow up interviews to some of the written responses during the task-based interview. All interviews were tape recorded and transcribed. Bell (1995) is of the view that for the sake of accuracy a tape recorder is a vital device, especially in interviews.

The collection of data was divided into three sessions as follows:

- ascertaining the student teachers' prior understanding of definitions;

- engaging them dynamically in the process of evaluating different given descriptions (definitions) for a rhombus; and

- assessing their own ability in evaluating other, new definitions for a rhombus.

\section{Clarification of Some Terminology}

This section is included in this paper because it contains definitions intended to be helpful in the analysis.

\section{The arbitrariness of mathematical definitions}

The arbitrariness of mathematical definitions refers to the existence (or choice) of different, alternative but correct definitions for the same concept.

For instance, when defining a trapezoid one can define it as a quadrilateral having at least one pair of opposite sides parallel. On the other hand, one can define it, if one wishes, as a quadrilateral having exactly one pair of opposite sides that are parallel. If you choose the first definition, a parallelogram is also a trapezoid. If you choose the second one, it is not. If the concept that definitions are arbitrary is well understood the above fact will not cause confusion, otherwise it might cause a great deal (see Vinner, 1991).

\section{Necessary and sufficient conditions}

For a condition in a given description (definition) to be sufficient, it must contain enough information (properties) to ensure that not only do we obtain the elements of the set we want to define, but only those elements (and not any others). However, normally we want to use as little information as possible, i.e. only as much as is really necessary.

\section{Correct definitions}

A description (definition) which contains conditions (properties) that are sufficient is said to be correct. In a correct definition, all the conditions may be necessary or some of the conditions may be unnecessary, i.e. it is possible to have unnecessary conditions in correct definitions.

\section{Incorrect definitions}

A definition is incorrect if it contains an incorrect property or if it contains insufficient properties.

\section{Incomplete definitions}

A definition is incomplete if it contains necessary but insufficient properties. So an incomplete definition is also an incorrect one.

\section{Economical and uneconomical definitions}

A correct definition can be either economical or uneconomical. An economical definition has only necessary and sufficient properties. It contains no superfluous information. On the other hand, an uneconomical definition has sufficient, but some unnecessary properties. In other words, it contains more information than necessary (redundant information).

\section{Rationale for use of the words economical and uneconomical}

It is generally accepted that definitions in mathematics should be minimal (economical). By this we mean that definitions should not contain parts which can be mathematically inferred from other parts of the definitions. For instance if one decides to define a 
rectangle in Euclidean geometry by means of its angles it is preferable to define it as a quadrilateral with three right angles and not as a quadrilateral having 4 right angles. This is because in Euclidean geometry, if a quadrilateral has 3 right angles one can prove that its fourth angle is also a right angle. So when formally defining the meaning of a term it is customary to give only the minimum required to understand the term.

This minimality principle is a crucial structural element of mathematics organised as a deductive system. In fact, it shapes the way in which mathematics progresses when it is presented deductively, namely after the definition, theorems which give you additional information about the concept are formulated and proved. Linchevsky et al (1992:54) made the following point in regard to definitions being minimal:

"If mathematical definitions were not minimal we would have to prove their consistency. For instance, if you define an equilateral triangle as a triangle whose sides are congruent and all its angles are congruent then you have to proceed by showing that these two properties can "live together." The most appropriate way of doing it in this case is to show that if all sides of a given triangle are congruent then its angles are also congruent. Therefore, what is the point of defining an equilateral triangle by both its sides and its angles if you should prove exactly the same theorems you would have to prove when going the minimal way? Being minimal is being economical".

However, there are a few cases in geometry where definitions are not minimal. A familiar one, perhaps, is the way in which some textbooks define congruent triangles. For example, "Congruent triangles are triangles which have corresponding sides congruent and corresponding angles congruent" (Gonin et al, 1974). We know, though, that it is sufficient to require less than that for two triangles to be congruent. This is expressed by each of the four congruence axioms.

\section{Data Analysis and Findings}

The analysis and findings will be presented in the context of each of the critical questions.

\section{Student Teachers' Prior Understanding of Definitions}

Two of the tasks used are given and discussed below.

\section{Task 1}

How would you describe what a rhombus is, over the phone, to someone who is not yet acquainted with it?

The students' responses were classified into the following categories

\begin{tabular}{|l|c|c|c|}
\hline Task 1 & $\begin{array}{c}\text { Incomplete or } \\
\text { wrong } \\
\text { definitions }\end{array}$ & $\begin{array}{c}\text { Uneconomical } \\
\text { definitions }\end{array}$ & $\begin{array}{c}\text { Economical } \\
\text { definitions }\end{array}$ \\
\hline $\begin{array}{l}\text { Group } \\
(\mathrm{N}=18)\end{array}$ & 4 & 13 & 1 \\
\hline
\end{tabular}

Here are some examples of incomplete or wrong definitions:

Letha: I would say a rhombus is a four-sided figure. It has both pairs of opposite sides equal. It also has both pairs of opposite sides parallel.

Xolisle: A rhombus is a parallelogram with four sides. Both opposite sides are equal and both opposite angles are equal and opposite sides parallel

These descriptions clearly contain too little information to guarantee the construction of a rhombus. These student teachers seem to view necessary conditions as sufficient conditions and appear to be operating only at Van Hiele Level 1.

Here are some examples of typical uneconomical definitions:

Mathunzi : I would tell him/her that it is a four sided, figure with all sides equal. If you join the opposite angles, the angle at the centre where the lines bisect each other will be a right angle and diagonals bisect the angles of the rhombus.

Ntombi: A rhombus is a four-sided figure, with diagonals bisecting each other at right angles and with each pair of opposite sides equal and parallel.

These student teachers provided more than the sufficient information required to construct a rhombus or to deduce the other properties from it, and therefore 
would appear to be operating at Van Hiele Level 2 (compare Burger \& Shaughnessy, 1986).

The following student teacher was the only one to provide a correct, economical definition:

Sifiso: I would say a rhombus is a closed foursided figure with all its sides equal.

Since this definition does not contain any redundant or superfluous information, it would appear that the student teacher is operating at Van Hiele Level 3.

\section{Task 2}

Which of the following descriptions do you think you would be able to use?

Circle these descriptions.

a. A rhombus is any quadrilateral with opposite sides parallel.

b. A rhombus is any quadrilateral with perpendicular diagonals.

c. A rhombus is any quadrilateral with two perpendicular axes of symmetry (each through a pair of opposite angles).

d. A rhombus is any quadrilateral with perpendicular, bisecting diagonals.

e. A rhombus is any quadrilateral with two pairs of adjacent sides equal.

f. A rhombus is any quadrilateral with all sides equal.

g. A rhombus is any quadrilateral with one pair of adjacent sides equal, and opposite sides parallel.

None of the student teachers selected the full complement of correct descriptions (definitions) namely c, d, f and g. However, three students selected only correct descriptions (definitions). For example, Mathunzi chose d and f, Owen chose g whilst Sanele chose $\mathrm{d}$ and $\mathrm{f}$.

The remaining 15 students chose at least one correct description (definition), although they also selected incomplete descriptions. For example:

- Twelve students selected: (a) A rhombus is any quadrilateral with opposite sides parallel.

- Eleven students selected: (e) A rhombus is any quadrilateral with two pairs of adjacent sides equal.
- Eight students selected: (b) A rhombus is any quadrilateral with perpendicular diagonals.

Their selection of incomplete descriptions (definitions) makes it clear that these student teachers don't understand that a description (definition) must contain necessary and sufficient properties. Whilst many students chose various options to describe a rhombus, none of the students chose the full complement of the correct descriptions, namely c, d, f, g. This therefore suggested that the students did not have a well-developed ability to evaluate definitions.

A single student teacher, Owen, chose only one description. The others, by choosing more than one, seemed to have at least an intuitive understanding of the arbitrary nature of definitions.

\section{Summary}

- The majority of the student teachers appeared to have an intuitive understanding of the arbitrary nature of definitions.

- Several student teachers understood a definition (description) of a given figure to be a list of properties of that given figure, which in fact is an uneconomical way to describe a figure (thus suggesting Van Hiele Level 2 understanding).

- Some student teachers incompletely define (describe) figures by viewing necessary conditions as sufficient conditions.

\section{Constructively evaluating different definitions for a rhombus}

The student teachers were next given a number of prerecorded Sketchpad scripts from De Villiers (1999), and asked the following main questions while playing the scripts:

a. Does the script construct a correct rhombus?

b. Which description in the table matches the script?

c. Drag the figure. Does it always remain a rhombus?

d. Is the given information sufficient for the construction of a rhombus? If yes/no, why?

e. Is all the given information necessary for the construction of a rhombus?

f. Is the description correct? If yes/no, why?

g. Is the description economical or uneconomical? Why? 


\section{Task 3}

One way of testing a description is to construct a figure complying with the description to see if it really gives the desired figure (we will use Sketchpad).

Open the scripts Rhombus 1.gss (Windows), and go on to check the descriptions a-g. Construct the appropriate givens required for each script, and click on the Step button repeatedly to make each script construct its figure. When the construction is finished, match each script with a description in the table. Drag the figure to see if it always remains a rhombus. In the table below, cross out the script names of any scripts that construct figures that are not always rhombuses.

\begin{tabular}{|l|l|}
\hline Script & Description (a-g) \\
\hline Rhombus 1 & \\
\hline Rhombus 2 & \\
\hline Rhombus 3 & \\
\hline Rhombus 4 & \\
\hline Rhombus 5 & \\
\hline Rhombus 6 & \\
\hline Rhombus 7 & \\
\hline
\end{tabular}

Although initially the student teachers required some guidance in constructing the required givens (prerequisite points) for each script, they quickly became independent as they progressed through the 7 scripts. Working through each script provided good learning opportunities for the student teachers to check whether the conditions for each script were sufficient to produce a rhombus. Due to space limitations, we shall here only discuss the script Rhombus 7. An example of an on-screen sketch produced by this script is shown in Figure 1 (though appearance may vary depending on the relative positions of the pre-requisite points $A$ and $B$ ).

When the construction was finished, the researcher firstly questioned the student teachers about the displayed lengths and gradients on the screen. The student teachers showed understanding of the displayed measurements. Upon asking the students whether the script constructed a correct rhombus, all responded that it was correct, apparently judging purely from a visual perspective. Only upon encouragement did the students check out their claim by measuring the sides to see if they were really all equal.

Furthermore, all 18 student teachers matched the script Rhombus 7 correctly to description g. The student teachers were then requested to drag the figure on Sketchpad and observe whether it always remained a rhombus. After the student teachers had dragged the figure around numerous times, they were confident

$$
\begin{aligned}
& \mathrm{m} \overline{\mathrm{AC}}=3.715 \mathrm{~cm} \\
& \mathrm{~m} \overline{\mathrm{AB}}=3.715 \mathrm{~cm} \\
& \text { Slope } \overline{\mathrm{AC}}=0.442 \\
& \text { Slope } \overline{\mathrm{DB}}=0.442 \\
& \text { Slope } \overline{\mathrm{AB}}=-1.291 \\
& \text { Slope } \overline{\mathrm{CD}}=-1.291
\end{aligned}
$$

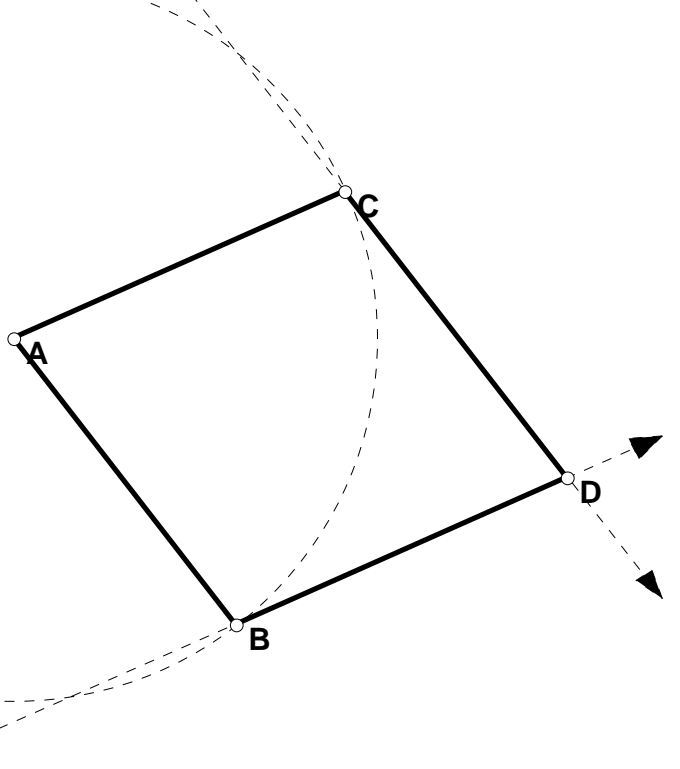

Figure 1: Example of an on-screen sketch 
that this figure always remained a rhombus. The student teachers also indicated that the given information was sufficient as well as necessary.

Some selected extracts from typical responses are given below:

Researcher: I now want you to look for a description in the list that fits in with Rhombus 7?

Xolisile:

g.

Researcher: Ok, right, $g$ is correct. I want you to focus on the definition or description there and I want you to tell me if the given information is sufficient for the construction of a rhombus?

Xolisile: Yes.

Researcher: Why?

Xolisile: The script always constructs a rhombus. If you drag it you always get a rhombus.

Researcher: Ok. Is the information necessary for the construction of a rhombus?

Xolisile: $\quad$ Yes, it is necessary.

All the student teachers identified it is a correct, economical definition because it contains necessary and sufficient conditions, which is indicative of Van Hiele Level 3 thinking.

Researcher: Is it an economical definition?

Xolisile: Yes.

Researcher: Why?

Xolisile: $\quad$ It is necessary and sufficient.

Researcher: Would you say that the definition is a correct definition of a rhombus?

Mathunzi: Ja, I would say it is a correct definition of a rhombus.

Researcher: Would you say it is a correct economical definition?

Mathunzi: Ja, it is economical.

Researcher: Why do you say economical?

Mathunzi: $\quad$ Aaaah. It is sufficient and necessary.

\section{Task 4}

List the descriptions from a-g that you think best describes a rhombus

Seventeen out of 18 students listed all four (i.e. c, $\mathrm{d}, \mathrm{f}$ and $\mathrm{g}$ ) correct descriptions as the ones that best describe the rhombus in Task 2. Only one student, Thandiwe, did not choose all four, but managed to identify three out of the four correct descriptions, namely c, $\mathrm{d}$ and $\mathrm{f}$.
Summary The student teachers' responses to Task 4, in comparison to their earlier responses to Task 2, clearly suggest the following as a result of being involved with the process of constructive evaluation of definitions in a dynamic geometry environment:

- The student teachers appear to have developed a deeper understanding of the arbitrary nature of definitions.

- They showed improved ability to select correct alternative definitions for a rhombus.

- The scripts and the use of dynamic dragging provided the student teachers with the opportunity to check whether the conditions in the given definitions were sufficient for the accurate construction of a rhombus.

- As a consequence, the student teachers exhibited a better understanding of when conditions are:

- Necessary and sufficient

- Necessary but not sufficient

- Sufficient but not necessary (and therefore also some ability to distinguish between economical and uneconomical definitions)

\section{Student teachers' competency to assess other definitions after the preceding process}

Task 5, consisting of 2 items, was utilised to determine how competent the students were in evaluating other possible definitions for rhombi after the preceding learning experience. It was given to the students immediately after all the interviews were completed. This task comprised only written responses which were done by all the students at one sitting, though all the students had access to Sketchpad whilst answering these questions. A discussion of one of the items from Task 5 is provided below.

\section{Task 5}

Consider the following definition:

"A rhombus is any quadrilateral with all sides equal, opposite sides parallel, and perpendicular and bisecting diagonals".

a. What are your comments about this definition?

b. Is it a good definition?

c. If not, how would you change it?

This item gives a correct but uneconomical description of a rhombus and therefore was designed 


\section{Rajendran Govender, Michael de Villiers}

to test the students' ability to recognise that the description is correct, but uneconomical (i.e. contains more information than necessary).

Fourteen out of 18 of the respondents recognised that this description was uneconomical (sufficient but has unnecessary properties). This item was also implicitly intended to evaluate the students' understanding of a "good" definition and it was encouraging to note that all fourteen students indicated that the description was not a good definition. The following are typical examples:

Jwara's response:

a. Correct definition. But it is sufficient and unnecessary.

b. No.

Fikile's response:

a. This is a correct uneconomical definition because it includes unnecessary properties and can be made economical in a number of ways .

b. No, it is not a good definition.

Sifiso's response:

a. This definition is correct but it also contains unnecessary information therefore it is uneconomical.

b. No, because it is uneconomical.

However, not all fourteen who identified it as an uneconomical definition gave clear responses. Indeed some showed either a measure of confusion or difficulty in clear formulation. Extracts from these four responses are now presented and discussed individually.

Mthembu's response:

a. The definition is correct but some of the information is not there (uneconomical).

b. No.

Though Mthembu says it is uneconomical, he says "some of the information is not there". This may suggest that he is actually operating at Van Hiele Level 2, and might prefer more properties (rather than less) in the definition, rather than simply not understanding the meaning of the term uneconomical.

Letha's response: a. The definition is uneconomical for a rhombus. It is insufficient but necessary.

b. No.

Similarly, Letha stated that it was uneconomical, but then contradicts himself by claiming that the information is insufficient. It is difficult to ascertain whether the difficulty is conceptual or just a matter of a minor confusion of correct mathematical terminology.

Siyande's response:

a. Uneconomical because the definition is insufficient.

b. No.

Just like Letha there is a contradiction in this statement as a definition cannot be (correct) economical, as well as insufficient. It appears that Siyande might have been reasoning as follows: If the properties are more than sufficient then it means that it is "not sufficient" which he then equivalently matched with the word "insufficient". However, without further interviewing it is impossible to determine whether the problem is conceptual, linguistic or a mixture of both.

Here are some examples of the four responses that did not identify the definition as uneconomical:

Innocent:

a. This is a good definition. In fact it is a correct economical definition as it contains necessary and sufficient conditions.

b. Yes.

Ephraim:

a. I think this definition is necessary and sufficient because it include all the properties of a rhombus.

b. Yes.

Ntuli:

a. Economical correct, definition contains necessary $\&$ insufficient conditions.

b. No.

(Ntuli may have thought it was not a good definition because he personally preferred another correct definition)

Item 5(c) was intended to assess whether the student teachers could provide a shorter, correct definition of a rhombus. Nine of the students came up with shorter, correct economical descriptions by 
leaving out some properties. Six of these students chose to define the rhombus in terms of its sides (a fairly standard textbook definition) as follows: $A$ rhombus is any quadrilateral with all sides equal.

Two students preferred to define a rhombus as a parallelogram with one pair of adjacent sides equal (which is also used in some textbooks). For example: A rhombus is any quadrilateral with one pair of adjacent sides equal, and opposite sides parallel.

However, Sboniso defined a rhombus just in terms of its diagonals (a seldom used definition in textbooks). For example: A rhombus is any quadrilateral with perpendicular bisecting diagonals.

Six students came up with different shorter versions, which were either still uneconomical, or insufficient, or contained an incorrect property. For example:

Uneconomical: Mthembu for example gave a definition that was correct, but still uneconomical: $A$ rhombus is any quadrilateral with both pairs of opposite sides equal and parallel and with diagonals bisect each other perpendicularly.

Note that the definition would have been economical (and a non-standard definition) had Mthembu left out the condition that the diagonals bisected each other.

Ntombi's response: A rhombus is any quadrilateral with all sides equal, opposite sides parallel and perpendicular, bisecting diagonals, forming two axes of symmetry.

Ntombi seemed to only list all the properties of a rhombus she knew, making it even more uneconomical. Her reasoning appears to be at Van Hiele Level 2.

Incomplete (Insufficient): Petros gave a definition that was insufficient. For example: A rhombus is any quadrilateral with opposite sides parallel.

Thandiwe also gave a definition that was insufficient. For example: A rhombus is any quadrilateral with all pairs of opposite sides parallel and bisecting diagonals.

However, it was encouraging to note that half the student teachers presented correct economical definitions in 5(c), which is reflective of $\mathrm{Van}$ Hiele Level 3 thinking.
Summary

- The majority of the student teachers were able to identify (realise) the description in Task 5 as correct but uneconomical, and half of them were able to change it to a correct economical description of a rhombus in 5(c).

- It would appear that this improved ability to evaluate and improve a given definition could in some measure be attributed to the earlier learning activities, i.e. the earlier construction, measurement and dragging activities with Sketchpad.

\section{Concluding Remarks}

The use of construction and measurement to evaluate the correctness of geometric statements (conjectures) before proofs are done is of course common practice among mathematical researchers. As a teaching approach it is also not new. For example, a similar approach was used effectively in the USEME teaching experiment during 1977/78 (see Human \& Nel et al, 1989). Similarly, Smith (1940) reported marked improvement in pupils' understanding of "if-then" statements by letting them first make constructions to evaluate geometric statements. In his research he found that it enabled pupils to learn to clearly distinguish between the "given condition(s)" and the "conclusion(s)", and laid the conceptual groundwork for an improved understanding of the eventual deductive proof.

However, this study is markedly different in that it took place within the context of dynamic geometry, where a geometric configuration can be continuously dragged into different shapes to check for invariance. Ideally, students should test geometric statements by making their own constructions within Sketchpad. However, since this requires a rather high level of technical knowledge of the software, it was decided to provide them with ready-made scripts that they could play through step by step and observe as the figure was gradually constructed. As the scripts are dependent on the arbitrary construction and positioning of the "given points", they sometimes produce crossed quadrilaterals which was a little confusing to some students. Accordingly, in the revised version of this activity (see De Villiers, in press) use will instead be made of the "Hide/Show" button facility of Sketchpad to produce figures step by step, ensuring that they all initially appear to be a rhombus. Only upon further dragging would students 
then be able to ascertain whether it always remains a rhombus, and therefore whether the conditions are really sufficient.

It should also be noted that since the dynamic geometry software provided conviction to all the student teachers, the role of the eventual deductive proofs (i.e. to prove the sufficiency of the definitions) was conceptualized as that of systematization rather than that of verification.

Although it was not a main focus of this study, the issue of hierarchical vs. partition definitions for a rhombus arose quite a few times while interviewing (or in discussion with) the student teachers. However, the dynamic nature of the rhombi constructed in Sketchpad seemed to make the acceptance of the hierarchical classification of a square as a special rhombus far easier than in a traditional non-dynamic environment, as the student teachers could easily drag the constructed rhombus until it became a square. This is, however, a matter for further research.

\section{Acknowledgement}

This research was partially funded by a National Research Foundation (NRF), Pretoria, South Africa grant from the Spatial Orientation \& Spatial Insight (SOSI) Project, coordinated by Prof. Dirk Wessels (UNISA), Dr. Hercules Nieuwoudt (PUCHE) and Prof. Michael de Villiers (UDW). The opinions and findings do not necessarily reflect the views of the NRF.

\section{References}

BELL, J., 1995, Doing Your Research Project. Buckingham: Open University Press

BURGER, W.F. \& SHAUGHNESSY, M., 1986, "Characterizing the Van Hiele levels of development in geometry” in Journal for Research in Mathematics Education, 17(1), pp. 31-48

DE VILLIERS, M., 1997, “The Future of Secondary School Geometry” in Pythagoras, 44, pp. 37-54

DE VILLIERS, M., 1998, "To teach definitions in geometry or teach to define?” in Olivier, A \& Newstead, K. (Eds.) Proceedings of 22nd PME- conference, University of Stellenbosch, 12-17 July 1998, Vol. 2, pp. 248-255

DE VILLIERS, M., 1999, Rethinking Proof with Geometer's Sketchpad, Key Curriculum Press, USA

DE VILLIERS, M. (In press), Rethinking Proof with Geometer's Sketchpad 4, Key Curriculum Press, USA

GONIN, A.A., ARCHER, I.J.M., SLABBER, G.P.L., LA REY NEL, G.D.E., 1974, “Congruent Triangles" in Modern Graded Mathematics For Standard 8 (Second Edition), Elsies River: National Book Printers Limited

GOVENDER, R., 2002, Student Teachers' Understanding \& Development of their Ability to Evaluate \& Formulate Definitions in a Sketchpad context, unpublished Master's Thesis, University of Durban-Westville

GRAVEMEIJER, K., 1994, "Educational Development and Educational Research" in Journal for Research in Mathematics Education, 25 (5): pp. 443-471

HUMAN, P. G. \& NEL, J. H. In co-operation with M. D. de Villiers, T. P. Dreyer and S. F. G. Wessels., (Eds.), 1989, USEME Teaching Experiment. Appendix A: Alternative Geometry Curriculum Material. RUMEUS Curriculum Material Series No. 11, University of Stellenbosch LINCHEVSKY, L., VINNER, S., KARSENTY, R., 1992, "To be or not to be minimal? Student teachers views about definitions in geometry" in Geeslin, W., Graham, K. (Eds.), Proceedings of the Sixteenth International Conference for the Psychology of Mathematics Education, Vol. 2, pp. 48 - 55. Durham, USA

Department of Education, 2002, National Curriculum Statement Grades 10 - 12 (Schools): Mathematics (Draft), Pretoria: Department of Education

SMITH, R. R., 1940, "Three major difficulties in the learning of demonstrative geometry" in The Mathematics Teacher, 33, pp. 99-134, 150-178

VINNER, S., 1991, "The Role of Definition in the Teaching and Learning of Mathematics” in D. Tall (Ed.), Advanced Mathematical Thinking, Kluwer Academic Publishers, pp. 65-81 